\title{
Las operaciones de información rusas en el conflicto del este de Ucrania
}

\section{Russia's information operations in the Eastern Ukraine conflict}

\section{RESUMEN:}

La caída de la URSS precipitó un cambio progresivo en las relaciones entre los países que habían formado parte del aparato estatal del gigante euroasiático. La evolución de este proceso, en el marco del espacio post-soviético, se ha encontrado significativamente marcada por la gestión de la información en los escenarios resultantes de los conflictos de interés surgidos a raíz de las disputas territoriales, que emergieron en el nuevo mapa de Europa del Este.

La cuestión nacional ucraniana ha sido uno de los capítulos protagonistas de las páginas de Internacional en los medios de comunicación a nivel global. Los hechos que han acontecido en torno a la guerra del Dombás, continúan siendo, hoy en día, un tema recurrente en la prensa. En este contexto, las operaciones de información han resultado un factor de especial relevancia a la hora de influir en el desarrollo de los acontecimientos, para favorecer una determinada agenda política y generar un estado de opinión que permita facilitar los movimientos estratégicos de los actores intervinientes.

La Federación de Rusia, como heredera del legado que dejó tras de sí la Unión Soviética, cuenta con numerosos intereses en la región oriental de Ucrania. Frente a su postura, se encuentra la política desplegada desde Kiev en los últimos años, que experimenta un paulatino acercamiento a la Unión Europea y al conjunto de Occidente. El presente trabajo estudia las técnicas de propaganda rusas aplicadas al conflicto ucraniano, el uso de la desinformación como táctica de guerra híbrida y su posible efectividad, de acuerdo con el estatus actual de las autoproclamadas repúblicas de Donetsk y Lugansk.

\section{PALABRAS CLAVE:}

Operaciones de información; Propaganda; Rusia; Ucrania; Guerra híbrida; Desinformación.

\section{ABSTRACT:}

The fall of the USSR generated a progressive change in the relations between countries that once were part of the state apparatus of the Eurasian giant. The evolution of this process, within the framework of the post-Soviet space, has been significantly marked by the management of information in the scenarios resulting from the conflicts of interest arising from territorial disputes, which emerged in the new map of Eastern Europe.

The Ukrainian national problem has been one of the main chapters of the International pages in the global media. The events that took place around the war in Dombass continue to be, today, a recurring theme in the press. In 
this context, information operations have been a factor of special relevance when it comes to influence the events, in order to favor a specific political agenda and generate a state of opinion that pushes the strategic movements of the actors involved.

The Russian Federation, as heir of the legacy left behind by the Soviet Union, has several interests in the Eastern region of Ukraine. On the other hand, there is the policy deployed from Kiev in recent years, which is in gradual approach to the European Union and the West. This paper studies the Russian propaganda techniques applied to the Ukrainian conflict, the use of fake news as a weapon of hybrid warfare and its possible effectiveness, according to the current status of the self-proclaimed republics of Donetsk and Lugansk.

KEY WORDS:

Information operations; Propaganda; Russia; Ukraine; Hybrid warfare; Fake news.

\section{Introducción}

\subsection{Los intereses de Rusia en Ucrania}

Las potencias occidentales desarrollan su acción exterior y juegan sus cartas en la arena geopolítica mirando de soslayo hacia el Este, recelosos de la actividad del gigante blanco que, desde Moscú, parece tratar de injerir en sus asuntos con la intención de preservar a toda costa sus intereses estratégicos. En el último lustro, la presunta intromisión del Kremlin en la vida política norteamericana ha sido un tema recurrente de análisis en los círculos académicos y periodísticos. A tales efectos, se ha marcado como núcleo -en torno al cual gravitan los eventos que conforman las relaciones entre los dos antiguos bloques- el tablero geográfico ucraniano.

Tras la desintegración de la URSS, Rusia se constituyó como la heredera del legado soviético y, como tal, asume su responsabilidad a la hora de mantener un estatus dominante en la región, especialmente si se trata de sostener un nivel de influencia hegemónico sobre las antiguas repúblicas soviéticas. El liderazgo de Boris Yeltsin quizás fue más cómodo en este sentido para el Oeste de lo que viene resultando el ejercido por Vladimir Putin desde su llegada al poder.

De este modo, atar las relaciones con Kiev es una prioridad para la Federación Rusa, cuyo caudal de relevancia mundial mana de dos fuentes complementarias a su dominio del espacio post-soviético: la membresía permanente en el Consejo de Seguridad de la ONU y el mantenimiento de su capacidad nuclear (Deyermond, 2014). En consecuencia, Moscú triangula estos tres factores para apuntalar una posición internacional que, en caso de depender de su competitividad económica, experimentaría una situación bien distinta.

De acuerdo con la profesora Ruth Deyermond, los tres elementos de supremacía han sido sometidos a la presión de Occidente en la última década. Iniciativas como los escudos de misiles de la OTAN en Centroeuropa y el empuje de Washington en dirección a la adhesión de Georgia y Ucrania a la OTAN han mantenido tensa la cuerda, especialmente desde el acercamiento de Kiev a la Unión Europea en 2014, con la firma del Tratado de Asociación entre ambos actores. La suma de los elementos citados supone, desde el prisma del gobierno de Moscú, una amenaza para la seguridad del Estado (Deyermond, 2014).

En línea con el punto anterior, en el mes de febrero de 2019 el expresidente Poroshenko firmó una enmienda constitucional para facilitar la incorporación de Ucrania a la OTAN y la Unión Europea antes de 2023 (RFE/RL, 19 de febrero de 2019). Ante el predecible escenario, Rusia comenzó a mover ficha años atrás con la anexión de Crimea y la desestabilización del 
territorio oriental ucraniano, asistiendo en diversas materias a los movimientos rebeldes prorrusos que, en el marco de la guerra civil aún por resolver, proclamaron las repúblicas populares de Donetsk y Lugansk. El siguiente paso vino tras el acceso de Volodymyr Zelensky a la presidencia de Ucrania, momento en el cual Moscú decidió conceder el pasaporte ruso a los habitantes de Dombás, lo cual dispara las alarmas en Kiev ante el miedo de «[...] que la emisión de pasaportes rusos pueda dar pie al futuro despliegue de tropas de Rusia en el territorio sublevado, con la excusa rusa de proteger a su gente», como ocurriera en la península de Crimea (Colás, 24 de abril de 2019).

Precisamente el uso de este recurso argumental - una suerte de diáspora del pueblo ruso en los que fueran territorios de su imperio - es uno de los mecanismos principales de la guerra de propaganda en la que se desenvuelve el Kremlin. Sin embargo, las operaciones de información buscan atraer, no sólo en el espacio post-soviético, sino donde exista posibilidad, una red de partidarios de la cosmovisión rusa, como base de apoyo ante las políticas de Estados Unidos, la Unión Europea y sus aliados, que confrontan sus intereses (Darczewska, 2014, p. 14).

\subsection{Metodología}

El marco metodológico empleado para esta investigación es el resultado de un examen hemerográfico del material periodístico relevante surgido a raíz de los acontecimientos que conforman la temática del objeto de estudio. La producción informativa derivada del asunto en cuestión -y los hechos que lo conforman-constituye un compendio de recursos documentales necesario para comprender la dimensión histórica, política y geográfica de la cuestión que nos ocupa, siendo estos aspectos los que dotan al contenido de su interés científico.

Este análisis comparativo ha incluido y tomado en consideración medios dispares y antagónicos desde el punto de vista narrativo y editorial, a efectos de contar con una perspectiva suficientemente amplia para revisar y contrastar las informaciones a través de las cuales se han extraído las consiguientes conclusiones.

La configuración de este método acude a un planteamiento hipotético-deductivo basado en la observación del fenómeno a través del testimonio de los actores informativos implicados. Estos últimos esgrimen un determinado discurso condicionado por un contexto geopolítico determinado y unos principios deontológicos subordinados al sesgo de sus intereses como parte del citado escenario. Una vez examinado el conjunto del relato desde sus perspectivas opuestas, es posible deducir las consecuencias e implicaciones en el ámbito material de la cuestión, confrontando dichos discursos con la realidad de los hechos acontecidos y su repercusión en el actual panorama internacional.

Esta metodología, dispone a su vez de un planteamiento semiótico, desde cuyo prisma se pretende realizar un análisis formal del discurso, interpretado y contextualizado en el cuadro de las relaciones internacionales marcadas por las agendas geoestratégicas de los antiguos bloques del este y el oeste, reeditadas y dirigidas por la política exterior de la Federación de Rusia, Estados Unidos, la Unión Europea, Ucrania y los países próximos a la frontera occidental rusa.

A partir de esta mecánica de estudio, se pretende concluir la importancia del mensaje propagandístico en dicho contexto, sus distintos usos, su proyección e instrumentalización como arma en uno de los conflictos de interés internacionales más notables de nuestro tiempo. 


\title{
2. Control reflexivo: propaganda y guerra híbrida
}

\author{
Según la doctora Snegovaya
}

\begin{abstract}
Rusia ha estado usando una avanzada forma de guerra híbrida que depende enormemente de un elemento de guerra informativa que los rusos denominan «control reflexivo". El control reflexivo empuja a un adversario más fuerte a elegir las acciones más beneficiosas para los objetivos rusos, mediante la modulación decisiva de la percepción del adversario respecto a la situación (Snegovaya, 2015, p. 7).
\end{abstract}

Autores como Javier Miguel-Gil, en referencia a esta particular manera de confrontar al adversario, apuntan a un encuadre estratégico denominado «doctrina Gerasimov» (Miguel-Gil, 2019, p. 112), aludiendo al jefe del Estado Mayor de las Fuerzas Armadas de la Federación Rusa.

Siguiendo este modus operandi, Moscú ha tratado de anticipar los movimientos del rival, presumiblemente basados en información considerada verdadera, para imitarlos y reflejarlos a través de una intensiva campaña de propaganda que persigue influenciar las determinaciones del oponente (Reid, 1987, p. 294). Esta técnica de espejo va acompañada de una metodología de repetición y respuesta ante los acontecimientos que, de acuerdo con Christopher Paul y Miriam Matthews, se centra en la diseminación de contenidos basados en la «interpretación de los sucesos emergentes que parecen favorecer sus temáticas y objetivos" (Paul y Matthews, 2016, p. 4).

Para alcanzar un nivel deseado de efectividad en sus operaciones de información, el gobierno ruso despliega una estrategia de medios multicanal que, no sólo abarca sus propios medios - financiados total o parcialmente por el Estado - , sino que hace un uso extensivo de las redes sociales e, incluso, se infiltra en medios de comunicación social a priori no afines, que pican el anzuelo de la desinformación orquestada. Probablemente su buque insignia en cuanto a difusión informativa sea el canal Russia Today, plataforma audiovisual que inició su andadura en 2005 con una inyección presupuestaria de 30 millones de dólares que, seis años después experimentaría un ascenso del 91,43\% hasta alcanzar los 350 millones en 2011 (Klimentov, 2013). Para el año 2014, la irrupción de Sputnik catapultaría la campaña informativa rusa con un plan de difusión de contenidos en 30 idiomas (Darczewska, 2004, pp. 11-12).

Se observa, por tanto, un extenso despliegue de medios que ya sugiere por sí sólo la importancia que otorga Moscú a las operaciones de información a la hora de generar confusión y enarbolar un discurso que mantenga a la opinión pública conectada a una hipotética visión alternativa de los hechos, descontaminada - siguiendo el argumentario esgrimido en estos medios - de la perspectiva unipolar que, según su criterio, pretende implantar Occidente en los medios de comunicación (Darczewska, 2004, pp. 11-12).

En definitiva, la difusión de material informativo -independientemente de su rigor- busca el enroque de los conflictos para ganar tiempo y, paulatinamente, obtener una posición de ventaja que pueda ser usada en otros escenarios geopolíticos. Los medios de comunicación financiados por el Estado ruso y, con presencia en occidente, sirven de apoyo para alcanzar este objetivo, cuya meta ulterior es facilitar una vieja táctica: «divide y vencerás». Para Rusia, favorecer la fragmentación territorial de un Estado satélite o antigua república soviética tam- 
poco es algo nuevo. Un ejemplo ilustrativo es su política respecto al problema enquistado de Transnistria, el Estado independiente de facto fuera del control del Gobierno moldavo.

El Kremlin no sólo auxilia a la región rebelde en materia militar, sino que proporciona hasta un $80 \%$ de su presupuesto y actúa como su representante internacional extraoficial. «Rusia también ha expedido alrededor de 150.000 pasaportes a residentes de la región, lo cual proporciona una justificación adicional a Moscú para interferir en los asuntos de Transnistria» (Popescu y Litra, 2012, p. 4).

\section{Los recursos argumentales}

El soporte o creación de un grupo poblacional afín a la agenda de intereses del Gobierno ruso es una figura que se repite. A nivel argumental, las catapultas de propaganda bajo el paraguas moscovita buscan dar visibilidad a la citada diáspora, situando el acento en la existencia de minorías rusas con un importante peso social y cultural en la realidad del país en cuestión. A tales efectos, podrán emplear este recurso narrativo para excusar las acciones y movimientos del Kremlin con respecto a Estados de su órbita. Mantener la primacía en las relaciones con los mismos, es una materia ante la cual la acción exterior rusa no está dispuesta a ceder terreno.

En el mantenimiento de una posición dominante respecto a su área geográfica de influencia, extender una política lingüística incisiva ha demostrado ser notablemente efectivo. Es bien sabido que el idioma es un arma arrojadiza típica de los nacionalismos, aunque, pese a todo, sigue resultando un recurso sorprendentemente útil. En su estrategia de guerra híbrida, Rusia ha empleado la defensa del lenguaje a capa y espada, para garantizar su influencia en Ucrania. Este hecho ha sido motivo de encendidos debates en la Rada de Kiev, desde donde se han tomado posturas de cierre de filas en torno al ucraniano, favoreciendo una huida de su ciudadanía rusófona hacia medios de comunicación amparados por el Kremlin, donde encuentran refugio a la vez que reciben el mensaje deseado por Moscú.

Esta táctica, al igual que las mencionadas previamente, no es novedosa, aunque sí ha sabido adaptarse a los tiempos actuales. Las plataformas de comunicación rusas y sus ramificaciones en el universo de Internet, son capaces de argumentar un discurso lingüístico como parte de la protección de una identidad nacional, hoy en día alejada de los postulados ideológicos de la Unión Soviética (Chivvis, 2017, p. 8). De esta manera, se articula un mensaje ciertamente más atractivo, que permite captar un mayor número de adeptos no sólo en Ucrania, sino en otras áreas como las repúblicas del Báltico, en las que el subterfugio lingüístico también se ha dejado ver. Por su parte, la deriva legislativa en tono restrictivo de países como Estonia y Letonia en lo referente a la diversidad lingüística de sus países, no hace sino aumentar la fractura étnica. La investigación de la profesora Hogan-Brun con respecto al tema ha señalado un importante factor de división, al relacionar la necesidad de dominio de las lenguas vernáculas bálticas para acceder al mercado laboral, especialmente en el sector público (Hogan-Brun, 2007, p. 577, citado en Best, 2013, p. 36).

El efecto péndulo generado por el rechazo del idioma ruso en Ucrania ha tendido un puente oportuno para que la Administración Putin intervenga visiblemente en la situación de Dombás. La táctica de control reflexivo ha facilitado una excusa para Moscú a la hora de 
posicionarse firmemente en la zona, poniendo por delante una retórica de defensa de los derechos humanos publicitada en sus medios de comunicación. A tales efectos Russia Today señalaba recientemente que

Con un burbujeante sentimiento anti-ruso en Kiev, el presidente Vladimir Putin ha lanzado un salvavidas a los residentes rusófonos del este de Ucrania aprobando un decreto para simplificar el proceso de obtención del pasaporte ruso. Las repúblicas separatistas de Lugansk y Donetks son el hogar de 3,7 millones de habitantes, predominantemente ruso parlantes (RT, 25 de abril de 2019).

A modo de síntesis, la estrategia comunicativa en el marco de las operaciones de información de Rusia - con especial incidencia en Ucrania - cimienta su discurso en base a la protección de la minoría étnica rusa. Para ello, amplifica los puntos de atención mediática en torno al blindaje de una lengua aislada de cuya situación desfavorable es posible obtener beneficio estratégico; así como de una auto atribuida custodia de los derechos de dicha población.

\section{Desinformación en la guerra de Dombás: el caso del vuelo «MH17»}

Las noticias falsas y el juego de la desinformación han sido - tanto en medios de comunicación como en redes sociales- claros protagonistas de los eventos que han rodeado el conflicto territorial ucraniano. Uno de los casos más significativos y trágicos fue el derribo del vuelo comercial «MH17». Este episodio, acontecido en 2014 y cuyas pesquisas han señalado recientemente a los primeros presuntos responsables, agregó un importante nivel de presión internacional sobre Rusia, poniendo en duda su imagen exterior y, desde luego, incrementando la desconfianza entre Bruselas y Moscú, debido a la más que sospechosa participación de elementos rusos en el marco del ataque (Hartman, Golovchenko y Augenstein, 2019).

Días después del siniestro, el jefe de la Dirección General de Asuntos Político-Militares de las Fuerzas Armadas de Rusia, teniente general Andrei Kartapolov, compareció ante los medios para exponer la versión oficial de la Federación Rusa ante las acusaciones de la comunidad internacional. En su presentación, el alto mando militar, proyectó lo que Toal y O'Loughlin han denominado «pruebas circunstanciales», destinadas a levantar la sombra de la duda en la opinión pública, culpando indirectamente al ejército ucraniano del incidente. Sin mencionar explícitamente la responsabilidad de Kiev, el teniente general mostró imágenes por satélite de sistemas de misiles antiaéreos Buk pertenecientes a las Fuerzas Armadas de Ucrania, dentro del rango de alcance del vuelo de Malaysia Airlines, arrojando la pregunta: «¿cuál era el propósito de desplegar tal cantidad de sistemas de defensa antiaérea cerca de Donetsk?» (Toal y O'Loughlin, 2017, p. 17).

Las alegaciones de Kartapolov fueron replicadas por Russia Today en sus ediciones en inglés y en castellano simultáneamente el mismo día de la rueda de prensa. Ambas informaciones, disponibles en sus sitios web, titulaban la noticia alegando la supuesta cercanía de un caza Su-25 ucraniano respecto al Boeing 777 malayo (RT, 21 de julio de 2014). Este dato, 
serviría de complemento para añadir otra incógnita en la búsqueda de alivio de responsabilidad rusa, generando confusión y devolviendo la pelota al campo de Kiev.

En este sentido, difuminar el padrinazgo militar del Kremlin sobre las milicias rebeldes constituyó una prioridad de la estrategia coordinada de medios propios con alcance en occidente. El 22 de julio de 2014, la edición en castellano de Russia Today continuaba con el hilo argumental del teniente general Kartapolov, lanzando en esta ocasión un recopilatorio de diez preguntas retóricas que cuestionaban las acusaciones de Estados Unidos e insinuaban la acción de fuerzas ucranianas en el desarrollo de los acontecimientos. Para ello, situó el punto de mira en los cuatro asuntos principales sobre los que Moscú sostenía su discurso (RT, 22 de julio de 2014):

- El desvío del vuelo «MH17» de su ruta original.

- La actividad de los radares militares ucranianos.

- El despliegue de defensas antiaéreas ucranianas.

- La presencia del Su-25 ucraniano.

A su vez, los cuatro bloques abrían dos líneas narrativas delimitadas. Los puntos uno y dos referían veladamente la implicación del Estado de Ucrania a la hora de manipular de forma malintencionada la trayectoria del vuelo, dentro del alcance de un misil tierra-aire de su pertenencia, lo cual explicaría - siguiendo esta lógica - el incremento de la actividad de determinados radares militares ucranianos - Kupol-M1 9S18- (RT, 22 de julio de 2014). En paralelo, los puntos tres y cuatro pretendían apuntar una voluntad y actividad hostil orquestada desde Kiev, hacia el área de Donetsk. En última instancia, esta actitud de Ucrania habría derivado en el derribo del aparato, por motivos sólo atribuibles a los movimientos de las fuerzas ucranianas, de acuerdo con la justificación defendida por Rusia.

Por su parte, después de cinco años de investigación, el Equipo Internacional Conjunto (JIT, por sus siglas en inglés) encargado del caso, concluyó en 2018 «que un misil Buk de la serie 9M38, llevado desde la base de la 53 Brigada Antiaérea del Ejército de la Federación Rusa en Kursk hasta la localidad ucrania de Donetsk, abatió el avión» (Ferrer, 2019). A pesar de las recusaciones de las milicias prorrusas, respaldadas por el Kremlin, los paramilitares de Donetsk habían confirmado la tenencia del mismo sistema de misiles a los medios rusos en el verano de 2014, e incluso publicaron en la red social $V K$, un comunicado informando sobre el derribo de lo que consideraron un avión militar An-26. Ante la evidencia del ataque a un vuelo de pasajeros, el aparato informativo ruso elaboró un mensaje culpando a la Fuerza Aérea Ucraniana (Colás, 17 de julio de 2019).

Tal y como recoge el corresponsal del diario El Mundo en Europa del Este, Xavier Colás,

a las 23.04 el canal $R T$ emitió un mensaje en nombre de un supuesto controlador aéreo español con el apodo en Twitter «spainbuca», supuestamente empleado del aeropuerto de Kiev Boryspil: «El Boeing era seguido por dos aviones de ataque ucranianos, y lo destruyeron». Poco después esta versión es recogida por Russia-24, por RIA Novosti, luego por ITAR-TASS y Channel One. Pero la persona detrás de la cuenta@spainbuca era José Carlos Barrios Sánchez, un estafador profesional residente en Rumanía con antecedentes penales. Así lo demostraron una investigación conjunta del portal de investigación RISE y el portal de información RFE/RL. Precisamente estos días El Confidencial ha difundido el contenido de una llamada en la que 
este embaucador admite haber recibido pagos del equipo de $R T$ a cambio de contar lo que ellos le pedían que dijese (Colás, 17 de julio de 2019).

Para aplacar el desconcierto generado a raíz de la campaña de propaganda rusa, el Gobierno de los Países Bajos creó un portal de transparencia respecto a la labor del JIT. La plataforma online incluye la explicación de las investigaciones forenses, policiales y militares, de cara al esclarecimiento de la tragedia y la depuración de responsabilidades.

A su vez, el portal de comunicación de la Fiscalía holandesa anunció la emisión de las órdenes internacionales de arresto contra los tres ciudadanos rusos y el ciudadano ucraniano señalados como presuntos implicados en el suceso². En contrapartida, Vladimir Putin compareció ante los medios y, de nuevo, la agencia Sputnik difundió sus declaraciones cuestionando las pruebas recabadas por el equipo de trabajo formado por expertos procedentes de Australia, Bélgica, Malasia y Ucrania (Sputnik, 20 de julio de 2019).

\section{Twitter y el estatus internacional de Crimea}

El uso de la red social Twittercomo plataforma de comunicación política dominante es, a día de hoy, una realidad consumada. En los últimos años, este servicio virtual ha adquirido la condición de publicación oficial en tiempo real, con la existencia a nivel global de perfiles verificados pertenecientes a organismos e instituciones en decenas de países. Los poderes del Estado han actualizado y adaptado sus actividades de comunicación pública a las herramientas que imponen tendencia, aprovechando el escenario global de «hiperconectividad» para contar con un mayor alcance en la difusión del mensaje hacia la opinión pública.

No sólo las figuras políticas emplean Twitter como altavoz ideológico, o los gobiernos como tablón de anuncios; incluso las autoridades religiosas han ajustado algunos de sus procedimientos a la difusión online a través de este instrumento (Kington, 2013). Sin embargo, en el caso que nos ocupa, las características de Twitter permiten ampliar aún más, si cabe, el nivel de intromisión de las redes sociales en el devenir de la realidad social actual.

En 2018, Twitter verificó la cuenta en su red social de la Oficina del Ministerio de Asuntos Exteriores de Rusia en Simferopol, inmiscuyéndose - aunque no fuera su intención - en el debate internacional sobre la legitimidad de la ocupación rusa de Crimea. La misión diplomática de Ucrania en el Reino Unido anunció la presentación de una queja formal ante la compañía estadounidense por este posicionamiento que, de una manera o de otra, valida ante la opinión pública la existencia de una delegación rusa no reconocida por Kiev (Brown, 2019). La postura del Gobierno ucraniano respecto a Crimea es, a su vez, respaldada por la comunidad internacional. En marzo de 2014 la Asamblea General de las Naciones Unidas instó a preservar la integridad territorial y soberanía nacional de Ucrania, adoptando una resolución con el propósito de solicitar a los países miembro el no reconocimiento de cambio alguno en el estatus político y legal de Crimea (ONU, 2014).

En contrapartida, la diplomacia rusa ha aprovechado la coyuntura para emitir mensajes desde un escenario dotado de mayor significación. Al hilo de la verificación de su perfil,

1/ Ver https://www.om.nl/mh17-ezine-juni2016/e-zine-en.html

2/ Ver https://www.om.nl/onderwerpen/mh17-crash/ 
@ PMSimferopol se ha encargado de recoger ejemplos de reconocimiento de Crimea como parte de Rusia en otros órdenes informativos. De este modo, trató de viralizar la errata de la $B B C$ al señalar como rusa la capital crimeana, explotando su nuevo atributo como «fuente fidedigna de información oficial respecto a Crimea», en palabras del Ministerio ruso de Exteriores (Brown, 2019).

La popularidad del perfil mantiene una tendencia ascendente que, con una media de entre cuatro y cinco publicaciones al día, ha experimentado un ascenso del $24,4 \%$ en los últimos ocho meses. A la luz de esta progresión, el departamento de Sergei Lavrov ha proclamado la popularidad de la cuenta entre los usuarios de Twitter (Brown, 2019). Asimismo, @ PMSimferopol ha centrado gran parte de su actividad en combatir el relato de Kiev, con tuits poniendo en duda la voluntad de resolución del conflicto por parte del lado ucraniano.

\#Ucrania no ha cumplido su obligación de retirar las tropas en el Dombás, de Donetsk y Lugansk. Representantes de la \#OSCE subrayaron que Kiev no ha mandado ninguna señal de preparación para comenzar la retirada de tropas. ¿Quién necesita realmente paz en la región? (Russia's MFA in Crimea, 14 de octubre de 2019a).

Seguidamente, el mismo día, la autoproclamada misión diplomática difundía imágenes de una manifestación en Kiev protagonizada por grupos radicales de extrema derecha, conmemorando el aniversario de la creación del Ejército de Insurgencia Ucraniano, milicia colaboracionista con la Alemania nazi. En el mismo tuit, anexaba una declaración condenatoria de dichos movimientos por parte de miembros del Congreso de los Estados Unidos ante la alarma de un creciente antisemitismo en Polonia y Ucrania (Russia's MFA in Crimea, 14 de octubre de 2019b). Los dos mensajes propagados prácticamente al unísono, con un fuerte apoyo visual, dieron la oportunidad a Rusia de situar en el mismo contexto una presunta actitud belicista por parte del Gobierno de Kiev, unida a una ideología totalitaria y antisemita con la que asociar al enemigo de cara a la opinión pública occidental, habida cuenta de que el mensaje se publicó en inglés.

\section{Conclusiones/Discusión}

La política de comunicación de la Federación Rusa respecto a Ucrania y, en general, a los conflictos geopolíticos que involucran a Occidente en algún ámbito de actuación, se basa en una estrategia de medios y omnipresencia en redes sociales destinada a la fidelización de un colectivo predispuesto a seguir la versión de la realidad promulgada desde Moscú. Durante la Guerra Fría, la «batalla por los corazones y las mentes» se centraba, principalmente, en la captación de adeptos al paquete ideológico de un bloque u otro. En esta pugna, la propaganda soviética jugó un papel determinante a la hora de intentar crear una quinta columna que pudiera desestabilizar los movimientos de los aliados en el lado oeste del telón de acero.

Desde la disolución de la URSS, se ha observado un progresivo giro argumental, especialmente notable tras la llegada de Putin al Kremlin. Prácticamente desde su acceso a la Presidencia, el mandatario ruso ha emprendido una lucha dialéctica fundamentada en el resurgimiento del poderío internacional soviético y, eventualmente, el intento de recuperar terreno, esta vez dejando de lado la iconografía comunista, para dar paso a una versión actualizada del Imperio Ruso. 
Sin embargo, en este episodio de la historia y las relaciones internacionales de Rusia, se ha impuesto la estrategia basada en la praxis de una guerra híbrida actualizada al panorama informativo y tecnológico contemporáneo. Para potenciar la consecución de sus objetivos, Moscú complementa su acción diplomática y militar con campañas de información multicanal y multilingüe destinadas, en gran parte, a ejercer ese control reflexivo concebido por los arquitectos de la propaganda soviética. De este modo, al atacar simultáneamente todas las ramas de un mismo frente, se fomenta un nicho de población favorable a sus intereses, tomando ventaja de divergencias políticas y diversidad de opiniones, para cultivar un relato con calado en la opinión pública, que condicione en su favor las decisiones de actores opuestos en un determinado escenario.

A la hora de poner en práctica esta política de disuasión, Rusia favorece la visión de sí misma como una alternativa opuesta a la hegemonía de Estados Unidos y la Unión Europea, tratando de señalar las debilidades y contradicciones de las democracias occidentales mientras exhibe un alarde político en sus medios de comunicación, caracterizados por una importante falta de autocrítica hacia el Kremlin. Así pues, tratando de sembrar una semilla narrativa que germine en el momento adecuado, Moscú y su aparato de medios recurre a viejos principios propagandísticos como la persuasión a través de la decepción, desdibujando realidades mediante desinformación o, combinando multitud de informaciones - verídicas o no-, para situar el acento argumental en el contexto deseado.

En el asunto particular ucraniano, el interés de Rusia pasa por mantener una parte del territorio de Ucrania bajo su paraguas de influencia. De esta manera, se fracciona el avance del país en su camino hacia la integración europea y, se sustenta un sector territorial que continúe actuando como tapón ante las ya perdidas repúblicas centroeuropeas, antaño países satélites de la Unión Soviética. Para ello, haciendo uso de su extensa red de medios y actividad en redes sociales, confronta el relato de Kiev, presentando al adversario como un Estado pérfido que elude sus responsabilidades respecto al mantenimiento de la paz y la conservación de la cultura rusa en la parte oriental del país. Además, valiéndose de su política de hechos consumados tras la anexión de Crimea y la injerencia en Dombás, proyecta un relato dirigido a legitimar su postura y posición respecto al curso de los acontecimientos y la resolución de la cuestión ucraniana, dando como fruto un resultado favorable a su agenda.

\section{Bibliografía}

BROWN, Hayes (14 de enero de 2019). "Twitter verified a Russian government account in Crimea and Ukraine is pissed», BuzzFeed News. Recuperado de https://www.buzzfeednews.com/article/ hayesbrown/twitter-russia-crimea-ukrane-verified

CHIVVIS, Christopher S. (2017). «Understanding Russian "Hybrid warfare" and what can be done about it». Rand Corporation Testimony, CT-468.

COLÁS, Xavier(17dejulio de 2019). «Cincoaños delMH17: cuatroacusadosyporfin un detenido». EIMundo. Recuperado de https://www.elmundo.es/internacional/2019/07/17/5d2ee5e321efa08d4c8b45c1.html

COLÁS, Xavier (24 de abril de 2019). «Vladimir Putin "regalará" pasaportes rusos en las zonas sublevadas del este de Ucrania». El Mundo. Recuperado de https://www.elmundo.es/internacional/ 2019/04/24/5cc0794321efa03c5f8b468a.html 
DARCZEWSKA, Jolanta (diciembre de 2014). «The information war on Ukraine: New challenges». Cicero Foundation Great Debate Paper, $n^{\circ}$ 14/08.

DEYERMOND, Ruth (27 de abril de 2014). "What are Russian's real motivations in Ukraine? We need to understand them». The Guardian. Recuperado de https://www.theguardian.com/ commentisfree/2014/apr/27/russia-motivations-ukraine-crisis

FERRER, Isabel (19 de junio de 2019). «Acusados del derribo del MH17 tres militares vinculados con la inteligencia rusa y un ucranio". El País. Recuperado de https://elpais.com/internacional/2019/06/19/ actualidad/1560937694_838543.html

HARTMANN, Mareike; GOLOVCHENKO, Yevgeniy y AUGENSTEIN, Isabelle (2019). «Mapping (dis-) information flow about the MH17 plane crash». ArXiv preprint arXiv:1910.01363. Recuperado de https://arxiv.org/abs/1910.01363

HOGAN-BRUN, Gabrielle (2007). «Language-in-education across the Baltic: Policies, practices and challenges». Comparative Education, 43(4), 557. Citado en BEST, M. (2013). «The ethnic Russian minority: A problematic issue in the Baltic States". Verges: Germanic and Slavic Studies in Review, 2 (1).

KINGTON, Tom (16 de julio de 2013). «Vatican offers 'time off purgatory' to followers of Pope Francis tweets». The Guardian. Recuperado de https://www.theguardian.com/world/2013/jul/16/vaticanindulgences-pope-francis-tweets

KLIMENTOV, Vassily (9 de julio de 2013). «RT, Russian soft power in images». Inaglobal. Recuperado de https://graph-memes-invites-talk-shows.inaglobal.fr/en/television/article/rt-russian-soft-powerimages

MIGUEL-GIL, Javier (2019). «El tratamiento informativo de la guerra híbrida de Rusia». URVIO, Revista Latinoamericana de Estudios de Seguridad, 25, 108-121.

ONU (27 de marzo de 2014). «General Assembly adopts resolution calling upon States Not to recognize changes in status of Crimea Region». GA/11493. Recuperado de https://www.un.org/press/ en/2014/ga11493.doc.htm

PAUL, Christopher y MATTHEWS, Miriam (2016). "The Russian "firehorse of falsehood" propaganda model». Perspective, PE-198-OSD.

POPESCU, Nicu y LITRA Leonid (2012). «Transnistria: a bottom-up solution». Brief policy. European Council on Foreign Relations.

REID, Clifford (1987). «Reflexive control in Soviet military planning». En B. DAILEY y P. PARKER. Soviet strategic deception (p. 294). Lexington, Estados Unidos, Lexington Books.

RFE/RL (19 de febrero de 2019). «Ukraine president signs constitutional amendment On NATO, EU membership». Radio Free Europe/Radio Liberty. Recuperado de https://www.rferl.org/a/ukrainepresident-signs-constitutional-amendment-on-nato-eu-membership/29779430.html

RT (21 de julio de 2014). «Ukrainian Su-25 fighter detected in close approach to MH17 before crash Moscow». Russia Today. Recuperado de https://www.rt.com/news/174412-malaysia-plane-russiaukraine/

RT (22 de julio de 2014). «Rusia: "Un avión de combate ucraniano volaba cerca del Boeing durante el siniestro"». Russia Today. Recuperado de https://actualidad.rt.com/actualidad/view/134648defensa-rusia-mh17-ucrania-avion

RT (25 de abril de 2019). «Ukraine passes controversial language law, isolating Russian-speakers». Russia Today. Recuperado de https://www.rt.com/news/457560-ukraine-language-law-russians/

Russia's MFA in Crimea (14 de octubre de 2019a). Tuit de @ PMSimferopol. Recuperado de https://twitter. com/PMSimferopol/status/1183673921079595008 
Russia's MFA in Crimea (14 de octubre de 2019b). Tuit de @ PMSimferopol. Recuperado de https://twitter. com/PMSimferopol/status/1183848438456373248

SNEGOVAYA, Maria (septiembre de 2015). «Putin's information warfare in Ukraine: Soviets origins of Russian hybrid warfare». Russia Report I.

SPUTNIK (20 de julio de 2019). «Russia: Vladimir Putin comments on JIT's MH17 report». Sputnik. Recuperado de https://www.youtube.com/watch?v=qpEr8QrNONw

TOAL, Gerard y O'LOUGHLIN, John (2017). «"Why Did MH17 crash?” Blame attribution, television news and public opinion in Southeastern Ukraine, Crimea and the De Facto States of Abkhazia, South Ossetia, and Transnistria». Territorial Conflicts After Communism. 